\title{
Importance des Parametres Hydrodynamiques dans la Repartition Spatiale des Sediments Superficiels des Plages de Limbe (Sud-Ouest Cameroun)
}

\author{
Kouekam Kengap Arnaud, M.Sc \\ Station Spécialisée de Recherche Halieutique et Océanographique de \\ Batoké, Limbé, Cameroun \\ Fowe Kwetche Paul Gustave, PhD \\ Togue Kamga Fulbert, PhD \\ Institut des Sciences Halieutiques, Douala, Cameroun \\ Ngueguim Jules Romain, PhD \\ Station Spécialisée de Recherche Halieutique et Océanographique de \\ Batoké, Limbé, Cameroun \\ Dingong Atoukoh Thérèse Ginette, M.sc \\ Institut des Sciences Halieutiques, Douala, Cameroun
}

Doi: 10.19044/esj.2019.v15n18p270 URL:http://dx.doi.org/10.19044/esj.2019.v15n18p270

\section{Résumé}

La présente étude contribue à la détermination de l'importance des paramètres hydrodynamiques agissant sur la côte de Limbe (Sud-ouest Cameroun) à l'aide de l'analyse granulométrique des sédiments superficiels des plages. Pour atteindre cet objectif, deux campagnes de prélèvements de sédiments respectivement sur les plages de Batoke et Debundscha ont été effectuées le long de 8 radiales perpendiculaires à la ligne de rivage, pour chaque radiale, trois prélèvements ont été effectués respectivement au niveau des trois unités géomorphologiques de la plage soit au total 24 échantillons ont été prélevés pour des analyses granulométriques et sédimentométries au laboratoire. Les principaux résultats obtenus nous montrent que les sédiments superficiels des plages de Batoke sont majoritairement caractérisés par des sédiments de la classe des sables fins, très bien classés et dont la distribution est presque symétrique vers les fins tandis que la plage de Debundscha est caractérisée par une prédominance des sédiments de la classe des sables moyens, très bien classés et dont la distribution est asymétrique vers les grossiers. Du point de vu hydrodynamique, cette répartition granulométrique des sédiments montre que les plages de Batoke et Debundscha sont majoritairement transportés par les houles à énergie constante dont les courants sont très faibles mais par rapport au site de Batoké, Debundscha 
présente des conditions de houle plus importante. Le paramètre de marnage évalué $(\mathrm{RTR}=3,01)$ supérieur à 1 durant la période d'étude montre que les courants de marée dominent sur les courants générés par la houle traduisant ainsi que la marée pourrait être le paramètre majoritairement responsable de la répartition spatiale des sédiments sur ces deux plages.

Mots-clés: Analyse granulométrique, sédiment, plage, unités morphologiques, Limbe

\title{
Importance of Hydrodynamic Parameters in the Spatial Distribution of Surface Sediments of the Limbe Beaches (South-Western Cameroon)
}

\author{
Kouekam Kengap Arnaud, M.Sc \\ Fisheries and Oceanography Research Station Batoke, Limbe, Cameroon \\ Fowe Kwetche Paul Gustave, PhD \\ Togue Kamga Fulbert, PhD \\ Institute of Fisheries and Aquatic Sciences, Douala, Cameroon \\ Ngueguim Jules Romain, PhD
}

Fisheries and Oceanography Research Station Batoke, Limbe, Cameroon

Dingong Atoukoh Thérèse Ginette, M.sc

Institute of Fisheries and Aquatic Sciences, Douala, Cameroon

\begin{abstract}
This paper contributes to the determination of the importance of the hydrodynamic parameters acting on the Limbe coast (South-West Cameroon) using the particle size analysis of surface sediments of the beaches. In achieving this objective, two sediment sampling campaigns on the beaches of Batoke and Debundscha respectively were carried out along 8 radials perpendicular to the shoreline. For each radial, three samples were taken respectively at the three geomorphological units. From the beach, a total of 24 samples were taken for particle size analysis and sedimentometry in the laboratory. The main results obtained show that the superficial sediments of the Batoke beaches are mainly characterized by sediments of the class of fine sands. They are very well classified and their distribution is almost symmetrical towards the end. On the other hand, the beach of Debundscha is characterized by a predominance of medium sands sediments. Also, they are
\end{abstract}


very well classified and their distribution is asymmetric towards coarse. From a hydrodynamic point of view, this granulometric distribution of sediments shows that Batoke and Debundscha beaches are mostly transported by constant energy swells with very low currents. Compared to the Batoke site, Debundscha has more severe swell conditions. The estimated tidal range $(\mathrm{RTR}=3.01)$ which is greater than 1 during the study period indicates that tidal currents dominate wave-generated currents. Thus, this indicates that the tide may be the only responsible parameter for the spatial distribution of sediment on the two beaches.

Keywords: Particle size analysis, sediment, range, morphological units, Limbe

\section{Introduction}

Aujourd'hui, plus de 50\% de la population mondiale habite dans des zones côtières, et l'érosion côtière est un phénomène bien connu le long des côtes de tous les pays du monde (Bird, 1985 ; Paskoff, 2001). Se référant aux travaux de la commission sur l'environnement côtier de l'Union Géographique Internationale, Bird (1985) estimait que le processus de recul du trait de côte affectait à l'époque plus de 70\% des plages de la terre. La morphologie des plages est fortement liée d'une part aux sources des sédiments qui les alimentent et d'autre part à l'énergie mise en jeu dans leur transport et leur dépôt. Les études granulométriques permettent de déterminer la relation entre les caractéristiques des sédiments (taille, nature) et leurs conditions de sédimentation (Ismail et al., 2015). Le déplacement des sédiments le long des côtes est lié essentiellement à l'hydrodynamique marine (Biagi, 1993), mais est également influencé par la granulométrie du sédiment (Sahu, 1964 ; Folk, 1966 ; Nordstrom, 1977 ; Pino \& Jaramillo, 1992 ; Aagaard, 2014). La nécessité de protéger les côtes contre l'érosion est devenue une préoccupation majeure. En effet, la grande partie des biens et services du monde se localise sur la frange littorale sous forte pression induite par la croissance galopante de la population avec pour corollaire l'augmentation des besoins (Adjoussi, 2001). Le Cameroun compte plusieurs plages parmi lesquelles celles de Limbe et Kribi. Plusieurs études ont été menées sur les plages de Kribi en ce qui concerne la répartition granulométrique des sédiments et le comportement des paramètres hydrodynamiques. A Limbe, peu d'informations sont disponibles concernant ces études, celles qui existent sont parfois obsolètes et fragmentaires. Les populations locales exercent plusieurs activités sur la côte à l'exemple du commerce, de la pêche, la construction des habitats et bien d'autres, celles-ci étant inconsciemment exposées aux phénomènes naturels tels que l'érosion côtière, le recul du trait de côte, les vents et vagues violentes, etc. La 
population lors de la pratique de ces activités quotidiennes doit être en sécurité et pour cela il serait judicieux que des études soient menées dans le but de renseigner la population, l'Etat et le touriste en ce qui concerne les conditions hydrodynamiques et environnementales auxquelles sont exposées les plages de la ville de Limbe et ses environs. Ainsi, pour une meilleure gestion des littoraux, en particulier le littoral Ouest camerounais, il est impératif de retracer les conditions hydrodynamiques responsables de la répartition spatiale des sédiments sur les plages de Limbe. De ce fait, l'objectif général de cette étude est d'apporter une contribution à la détermination de l'intensité des paramètres hydrodynamiques agissant sur la côte de Limbe à l'aide des analyses granulométriques des sédiments superficiels des plages. Plus spécifiquement, il s'agira d'évaluer la distribution spatiale des particules sédimentaires dans les différentes unités morphologiques des plages, de décrire les conditions hydrodynamiques en fonction de la distribution spatiale des particules sédimentaires des plages étudiées et de caractériser les paramètres de houles et de marées durant la période de l'étude.

\section{Matériel et méthode}

\section{Présentation du site d'étude}

Le site d'étude est situé au pied du Mont Cameroun entre les latitudes $04^{\circ} 02,031^{\prime}$ et $04^{\circ} 05^{\prime} 24.3^{\prime}$ ' $\mathrm{N}$ et longitudes $009^{\circ} 05.401^{\prime}$ 'et $008^{\circ} 59^{\prime} 31.5^{\prime}$ 'Est. Il est donc recouvert de coulées basaltiques issues de ses différentes éruptions. Les minéraux de ses roches sont hydrolysés par les eaux pluviales chaudes et abondantes, définissant un sol de type ferralitique (Muller \& Gavaud, 1979). Le climat régnant dans la zone d'étude est de type tropical côtier avec 02 saisons : une longue saison de pluie allant de février à novembre avec juillet le mois le plus pluvieux $(720 \mathrm{~mm})$ et une courte saison sèche allant de décembre à janvier avec mars le mois le plus chaud $\left(26,4^{\circ} \mathrm{C}\right.$ température moyenne) (Source : www. climate-data.org). 


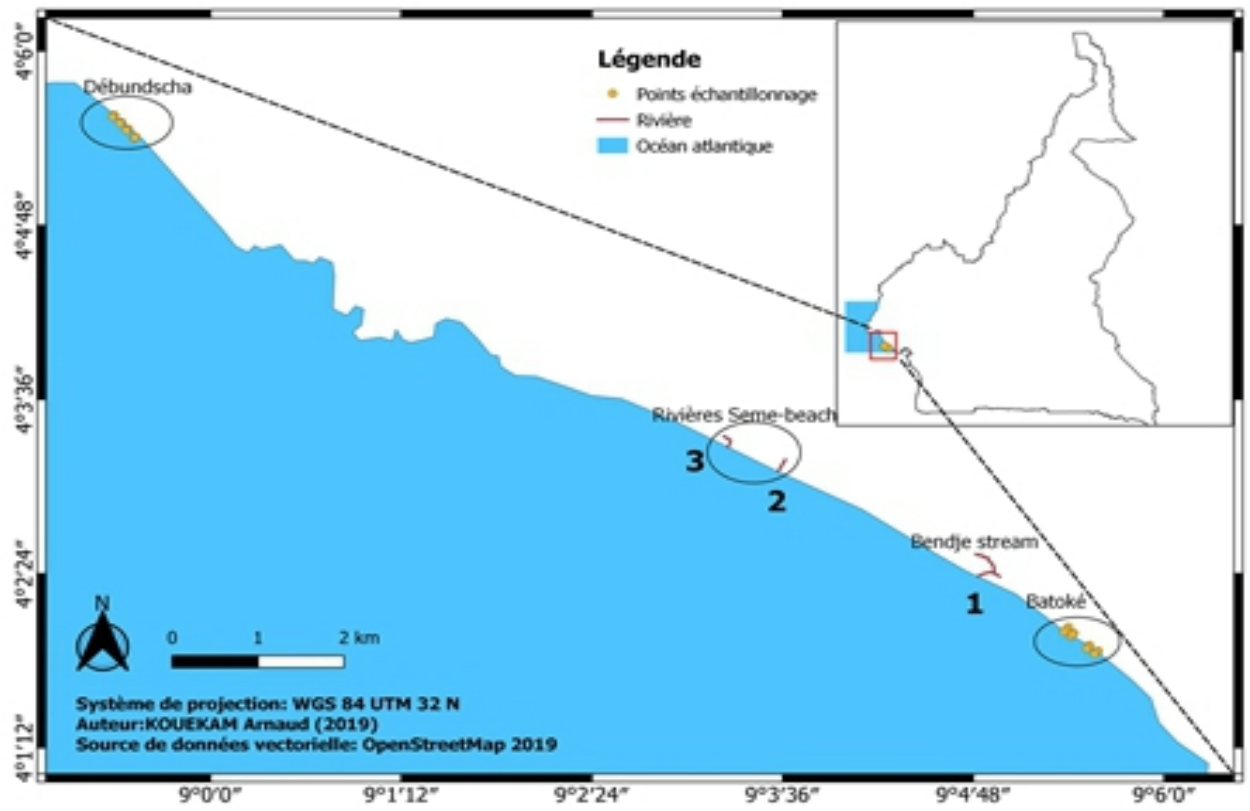

Figure 1. localisation de la zone d'étude et le réseau hydrographique de la localité

\section{Matériels}

Deux sites ont été retenus pour la réalisation de cette étude pour des raisons telles que l'accessibilité et la proximité de la zone et l'intérêt socioéconomique de la zone. Ainsi, deux campagnes de prélèvements des sédiments de surface sur les plages retenues ont été effectuées au cours de l'étude.

\section{Matériel d'échantillonnage sur le terrain}

Au cours de cette étude, des bouteilles en plastique de 1 litre de volume ont été utilisées, pour prélever les sédiments de surface dans les différentes unités morphologiques des plages étudiées et un récepteur GPS de marque GARMIN de la série MONTANA 650t a été utilisé pour la prise des coordonnées géographiques des différents points d'échantillonnages dont les différentes stations et leurs coordonnées sont reportées dans le tableau cidessous, l'échelle de marée de quatorze (14) décimètre $(\mathrm{dm})$ de long pour validation des données obtenues dans les sites internet www.surfforecast.com/breaks/Seme-Beach

et www.mareespeche.com/af/cameroon/bekumu de houle et de marée respectivement qui permettra d'évaluer le paramètre de marnage. 
Tableau 1. Coordonnées géographiques des différents points d'échantillonnage

\begin{tabular}{|c|c|c|c|c|}
\hline Sites & Radiales & Stations & Longitudes & Latitudes \\
\hline \multirow{24}{*}{ Batoke } & \multirow[b]{3}{*}{ Radiale 1} & Haut de plage 1 & $009^{\circ} 05.401^{\prime}$ & $04^{\circ} 02.031^{\prime}$ \\
\hline & & Estran 1 & $009^{\circ} 05.386^{\prime}$ & $04^{\circ} 02.014^{\prime}$ \\
\hline & & Bas de plage 1 & $009^{\circ} 05.373^{\prime}$ & $04^{\circ} 01.998^{\prime}$ \\
\hline & \multirow[b]{3}{*}{ Radiale 2} & Haut de plage 2 & $009^{\circ} 05.435^{\prime}$ & $04^{\circ} 01.989^{\prime}$ \\
\hline & & Estran 2 & $009^{\circ} 05.415$ & $04^{\circ} 01.965^{\prime}$ \\
\hline & & Bas de plage 2 & $009^{\circ} 05.425^{\prime}$ & $04^{\circ} 01.979^{\prime}$ \\
\hline & \multirow[b]{3}{*}{ Radiale 3} & Haut de plage 3 & $009^{\circ} 05.533^{\prime}$ & $04^{\circ} 01.905^{\prime}$ \\
\hline & & Estran 3 & $009^{\circ} 05.527^{\prime}$ & $04^{\circ} 01.895^{\prime}$ \\
\hline & & Bas de plage 3 & $009^{\circ} 05.516^{\prime}$ & $04^{\circ} 01.882^{\prime}$ \\
\hline & \multirow[b]{3}{*}{ Radiale 4} & Haut de plage 4 & $009^{\circ} 05.588^{\prime}$ & $04^{\circ} 01.867^{\prime}$ \\
\hline & & Estran 4 & $009^{\circ} 05.576^{\prime}$ & $04^{\circ} 01.858^{\prime}$ \\
\hline & & Bas de plage 4 & $009^{\circ} 05.565^{\prime}$ & $04^{\circ} 01.844^{\prime}$ \\
\hline & \multirow[b]{3}{*}{ Radiale 1} & Haut de plage 1 & $008^{\circ} 59^{\prime} 23.7^{\prime \prime}$ & $04^{\circ} 05^{\prime} 33.5^{\prime \prime}$ \\
\hline & & Estran 1 & $008^{\circ} 59^{\prime} 23.5^{\prime \prime}$ & $04^{\circ} 05^{\prime} 33.2^{\prime \prime}$ \\
\hline & & Bas de plage 1 & $008^{\circ} 59^{\prime} 23.1^{\prime \prime}$ & $04^{\circ} 05^{\prime} 32.8^{\prime \prime}$ \\
\hline & \multirow[b]{3}{*}{ Radiale 2} & Haut de plage 2 & $008^{\circ} 59^{\prime} 26.4^{\prime \prime}$ & $04^{\circ} 05^{\prime} 30.6^{\prime \prime}$ \\
\hline & & Estran 2 & $008^{\circ} 59^{\prime} 26.1^{\prime \prime}$ & $04^{\circ} 05^{\prime} 30.4^{\prime \prime}$ \\
\hline & & Bas de plage 2 & $008^{\circ} 59^{\prime} 25.8^{\prime \prime}$ & $04^{\circ} 05^{\prime} 30.1^{\prime \prime}$ \\
\hline & \multirow[b]{3}{*}{ Radiale 3} & Haut de plage 3 & $008^{\circ} 59^{\prime} 29.0^{\prime \prime}$ & $04^{\circ} 05^{\prime} 27.8^{\prime \prime}$ \\
\hline & & Estran 3 & $008^{\circ} 59^{\prime} 28.7^{\prime \prime}$ & $04^{\circ} 05^{\prime} 27.5^{\prime}$ \\
\hline & & Bas de plage 3 & $008^{\circ} 59^{\prime} 28.3^{\prime \prime}$ & $04^{\circ} 05^{\prime} 27.2^{\prime \prime}$ \\
\hline & \multirow[b]{3}{*}{ Radiale 4} & Haut de plage 4 & $008^{\circ} 59^{\prime} 31.7^{\prime}$ & $04^{\circ} 05^{\prime} 24.6^{\prime \prime}$ \\
\hline & & Estran 4 & $008^{\circ} 59^{\prime} 31.1^{\prime}$ & $04^{\circ} 05^{\prime} 23.9^{\prime \prime}$ \\
\hline & & Bas de plage 4 & $008^{\circ} 59^{\prime} 31.5^{\prime \prime}$ & $04^{\circ} 05^{\prime} 24.3^{\prime \prime}$ \\
\hline
\end{tabular}

\section{Matériel de Laboratoire}

Les différents échantillons prélevés sur le terrain ont fait l'objet d'analyse au laboratoire et pour cela, une étuve a été utilisée pour sécher les échantillons, une balance électronique a été utilisé pour peser les échantillons séchés, une colonne de 10 tamis de type AFNOR de $0,1 \mathrm{~mm}$ à $5 \mathrm{~mm}$ de diamètre $(0,1 \mathrm{~mm} ; 0,16 \mathrm{~mm}, 0,2 \mathrm{~mm} ; 0,315 \mathrm{~mm} ; 0,4 \mathrm{~mm} ; 0,63 \mathrm{~mm} ; 0,8 \mathrm{~mm}$; $1 \mathrm{~mm} ; 2 \mathrm{~mm}$ et $5 \mathrm{~mm}$ ) a été utilisés pour tamiser les échantillons séchés, un vibrotamis actionné avec du courant électrique pour les opérations de tamisage, des assiettes métalliques pour conserver les sédiments, une éprouvette en plastique graduée de 1 litre pour l'analyse de sédimentométrie, un densimètre gradué de 0 à 60 de forme torpille, de l'eau déminéralisée, un thermomètre de mercure 0 à $50^{\circ} \mathrm{C}$ gradué en cinquièmes de degré, un 
chronomètre pour prendre les différentes durées de temps et une solution défloculante (solution d'héxamétaphosphate de sodium à 5\%).

\section{Méthode}

L'échantillonnage a été effectué, au mois d'avril 2018, le long de 8 radiales perpendiculaires à la ligne de rivage (4 radiales sur la plage de Batoke et 4 sur la plage de Debundscha). Pour chaque radiale, trois prélèvements ont été effectués respectivement au niveau des trois unités géomorphologiques de la plage depuis le haut de plage jusqu'au bas de plage soit au total 24 échantillons prélevés. Chaque échantillon de sédiments a été prélevé sur une surface de $4000 \mathrm{~m}^{2}$ (rectangle de $100 \mathrm{~m} \mathrm{x} 40 \mathrm{~m}$ ) et sur une épaisseur d'environ $5 \mathrm{~mm}$ couvrant ainsi toute la couche active (Chauhan, 1992 ; Abuodha, 2003). Les échantillons prélevés ont fait l'objet de divers traitements au laboratoire de géotechnique de l'Ecole Nationale Supérieure Polytechnique de Yaoundé. Ces échantillons prélevés ont fait l'objet de deux analyses (par tamisage à sec et par sédimentométrie).

Ils ont été mis dans des assiettes métalliques, séchés à l'étuve pendant 24 heures à $105^{\circ} \mathrm{C}$, des échantillons ainsi séchés, $1000 \mathrm{~g}$ ont été prélevé pour effectuer le tamisage et les différents refus et passants ont été pesés et enregistrés dans un tableau. En ce qui concerne la sédimentométrie effectuée après tamisage à sec, l'essai a été fait sur $20 \mathrm{~g}$ de sédiments prélevés des passants, ensuite $30 \mathrm{~cm}^{3}$ de solution défloculante ont été versé dans une éprouvette en plastique d'1 litre à laquelle $200 \mathrm{~cm}^{3}$ d'eau déminéralisée ont été ajouté puis les $20 \mathrm{~g}$ de sédiments ont été inséré tout en imbibant l'ensemble pendant 24h. Par la suite l'ensemble a été soumis à une agitation mécanique pendant 5 minutes puis l'eau déminéralisée a été complété à 1 litre dans l'éprouvette. Ensuite, les différentes éprouvettes ont été mises dans un bain d'eau à température constante à $20^{\circ} \mathrm{C}$. Les différents paramètres de cet ensemble ont été pris à l'aide d'un densimètre et d'un thermomètre pendant des intervalles de temps bien précis (30 secondes, 1, 2, 3, 5, 10, 30 minutes, 1, 2, 3, 5, 24 heures) et enregistrés dans un tableau afin d'effectuer les différents calculs et faire des analyses selon les paramètres étudiés (Folk \& Ward, 1957).

\section{Résultats et Discussion}

\section{Résultats}

Evaluation de la distribution spatiale des particules sédimentaires dans les différentes unités morphologiques des plages de Batoke et Debundscha a) Courbes cumulatives des sédiments superficiels des plages de Batoke et Debundscha

Les calculs effectués à partir des résultats obtenus au laboratoire ont permis d'obtenir les courbes cumulatives ci-après (Figure2 1\&2). Ces courbes montrent l'évolution des grains en fonction des pourcentages de tamisât des 
différents échantillons sur chaque plage. Il en ressort que ces sédiments à travers ces courbes représentent une distribution spatiale et une évolution granulométrique hétérogènes.
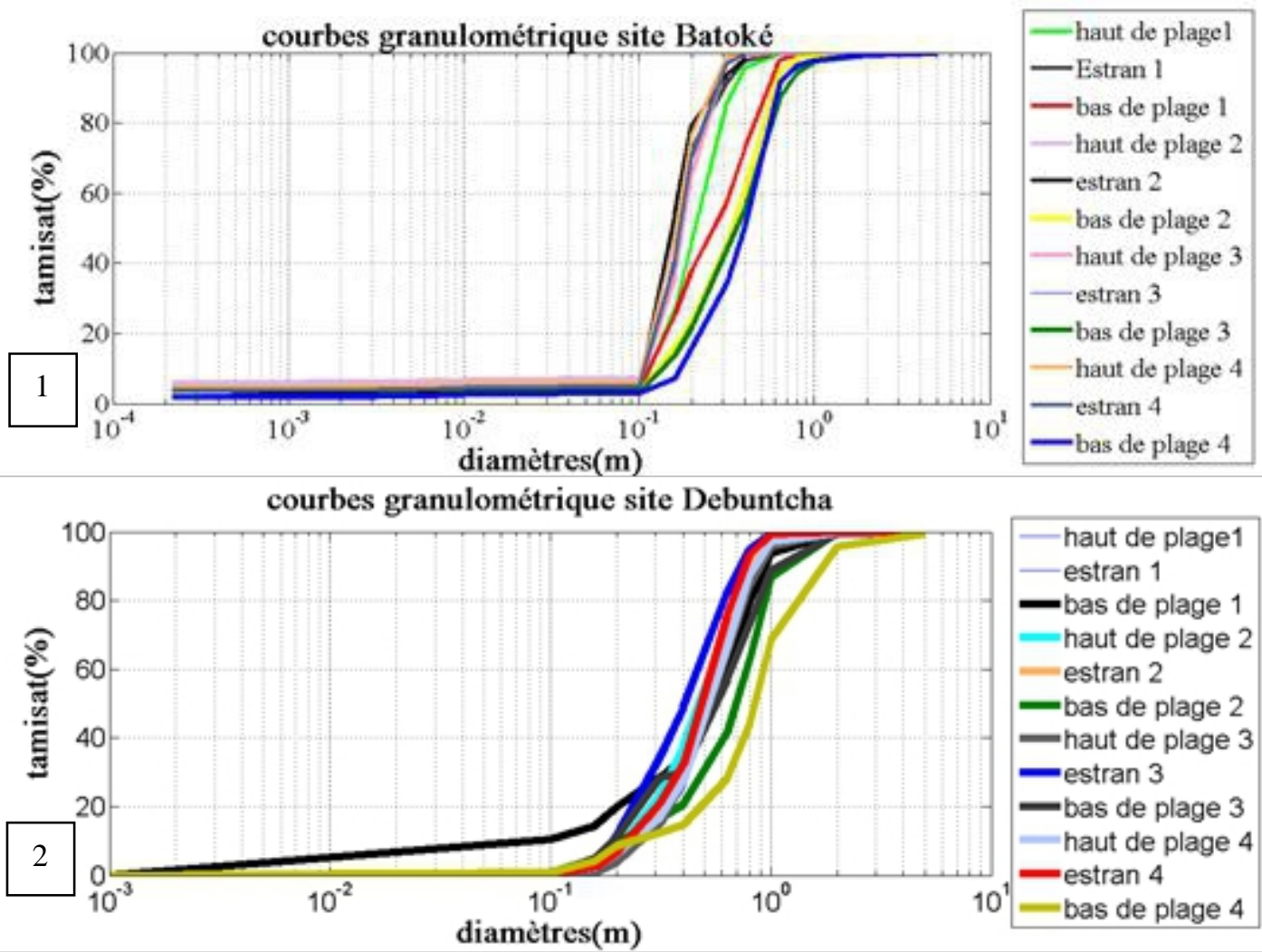

Figure 2. Courbes granulométriques des plages de Batoke (1) et de Debundscha (2)

\section{b) Paramètres granulométriques étudiés sur les plages de Batoke et Debundscha}

Le calcul des paramètres granulométriques des deux sites est résumé dans le tableau ci-dessous :

Tableau 2. Paramètres granulométriques calculés du site de Batoke

\begin{tabular}{|l|l|l|l|l|l|}
\hline $\begin{array}{l}\text { Unités } \\
\text { morphologiques }\end{array}$ & Moyenne & Sorting & Skewness & $\begin{array}{l}\text { Coefficient } \\
\text { d'acuité }\end{array}$ & $\begin{array}{l}\text { Coefficient } \\
\text { d'uniformité }\end{array}$ \\
\hline Haut de plage 1 & 2,25 & $-0,549$ & 0,0332 & 0,933 & 1,76 \\
\hline Haut de plage 2 & 2,53 & $-1,01$ & $-0,29$ & 3,12 & 1,636 \\
\hline Haut de plage 3 & 2,47 & $-0,481$ & 0,126 & 0,918 & 1,5 \\
\hline Haut de plage 4 & 2,58 & $-0,481$ & $-0,03$ & 1,222 & 1,417 \\
\hline Estran 1 & 2,55 & $-0,602$ & 0,189 & 1,265 & 1,545 \\
\hline Estran 2 & 2,62 & $-0,463$ & 0,17 & 1,413 & 1,545 \\
\hline Estran 3 & 2,51 & $-0,451$ & 0,141 & 0,976 & 1,5 \\
\hline Estran 4 & 2,53 & $-0,529$ & 0,001 & 0,949 & 1,619 \\
\hline Bas de plage 1 & 2,075 & $-0,793$ & $-0,278$ & 0,727 & 2,909 \\
\hline Bas de plage 2 & 1,70 & $-0,824$ & $-0,22$ & 0,83 & 3 \\
\hline
\end{tabular}




\begin{tabular}{|l|l|l|l|l|l|}
\hline Bas de plage 3 & 1,55 & $-0,871$ & $-0,168$ & 0,881 & 3 \\
\hline Bas de plage 4 & 1,51 & $-0,754$ & $-0,329$ & 0,971 & 2,5 \\
\hline
\end{tabular}

Tableau 3. Paramètres granulométriques calculés du site de Debundscha

\begin{tabular}{|l|l|l|l|l|l|}
\hline $\begin{array}{l}\text { Unités } \\
\text { morphologiques }\end{array}$ & Moyenne & Sorting & Skewness & $\begin{array}{l}\text { Coefficient } \\
\text { d'acuité }\end{array}$ & $\begin{array}{l}\text { Coefficient } \\
\text { d'uniformité }\end{array}$ \\
\hline Haut de plage 1 & 1,137 & $-0,642$ & $-0,207$ & 1,02 & 2,304 \\
\hline Haut de plage 2 & 1,113 & $-0,743$ & $-0,167$ & 0,904 & 2,5 \\
\hline Haut de plage 3 & 0,96 & $-0,658$ & $-0,191$ & 1,057 & 2,143 \\
\hline Haut de plage 4 & 1,02 & $-0,674$ & $-0,237$ & 1,108 & 2,458 \\
\hline Estran 1 & 0,963 & $-0,647$ & $-0,297$ & 1,092 & 2,346 \\
\hline Estran 2 & 1,08 & $-0,65$ & $-0,281$ & 1,187 & 2,478 \\
\hline Estran 3 & 1,383 & $-0,7302$ & $-0,153$ & 0,794 & 2,4 \\
\hline Estran 4 & 1,13 & $-0,641$ & $-0,268$ & 1,006 & 2,455 \\
\hline Bas de plage 1 & 1,26 & $-1,482$ & $-0,421$ & 1,642 & 7,011 \\
\hline Bas de plage 2 & 0,781 & $-0,887$ & $-0,440$ & 1,311 & 3,714 \\
\hline Bas de plage 3 & 1,017 & $-0,959$ & $-0,287$ & 0,855 & 3,35 \\
\hline Bas de plage 4 & 0,353 & $-0,978$ & $-0,230$ & 1,514 & 3,869 \\
\hline
\end{tabular}

\section{Moyenne}

Les données de moyenne obtenues à Batoke (Tableau 2) montrent diverses classes granulométriques en fonction des unités morphologiques étudiées. Ainsi, le haut de plage est constitué des grains compris entre 2,25ø et $2,587 \varnothing$, l'estran de $2,513 \varnothing$ et 2,623ø et le bas de plage entre $1,517 \varnothing$ et 2,077 $\varnothing$. Ces résultats montrent que le site de Batoké est majoritairement constitué des sables fins $(2 \varnothing<\mathrm{Mz}<3 \varnothing$ ou $125 \mu \mathrm{m}<\mathrm{Mz}<250 \mu \mathrm{m})$ avec une infime proportion de sable moyen à certains endroits de la plage $(1 \varnothing<\mathrm{Mz}<2 \varnothing$ ou $250<\mathrm{Mz}<500$ $\mu \mathrm{m})$.

La moyenne des grains au niveau de Debundscha (Tableau 3) est comprise entre $0,96 \varnothing$ et $1,137 \varnothing$ sur le haut de plage, entre $0,963 \emptyset$ et $1,383 \varnothing$ sur l'estran et entre $0,353 \varnothing$ et $1,26 \varnothing$ au niveau du bas de plage. Ces résultats montrent que le site de Debundscha est constitué majoritairement des sables moyens $(1 \varnothing<\mathrm{Mz}<2 \varnothing$ ou $250<\mathrm{Mz}<500 \mu \mathrm{m})$ avec une infime proportion de sable grossier à certains endroits de la plage $(\mathrm{Mz}<1 \varnothing$ ou Mz $>500 \mu \mathrm{m})$. 


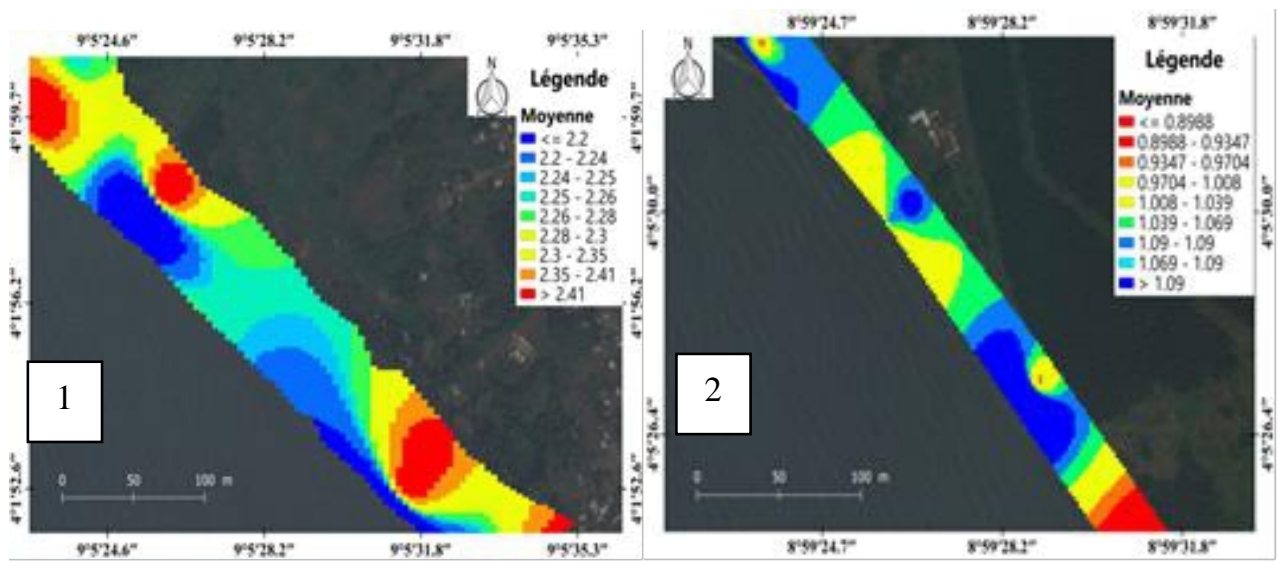

Figure 3. Distribution de la taille moyenne des grains au niveau de la plage de Batoke (1) et au niveau de la plage de Debundscha (2).

\section{Indice de classement (So-sorting)}

Les distributions granulométriques obtenues à Batoke (Tableau 2) montrent que les indices de classement de haut de plage se situent entre $-1,01 \varnothing$ et $-0,481 \varnothing$, ceux de l'estran entre $-0,602 \varnothing$ et $-0,451 \varnothing$ et ceux du bas de plage entre $-0,871 \varnothing$ et $-0,754 \varnothing$. Ces résultats montrent que les sédiments superficiels de cette plage sont des sables très bien classés $(\sigma<0,35 \varnothing)$.

Les distributions granulométriques obtenues à Debundscha (Tableau 3 ) montrent que les valeurs du Sorting du haut de plage se situent entre $-0,743 \varnothing$ et $-0,642 \varnothing$, ceux de l'estran entre $-0,7302 \varnothing$ et $-0,641 \varnothing$ et ceux du bas de plage entre $-1,482 \emptyset$ et $-0,887 \varnothing$. Ces résultats montrent que les sédiments de cette plage sont des sables très bien classés $(\sigma<0,35 \varnothing)$.

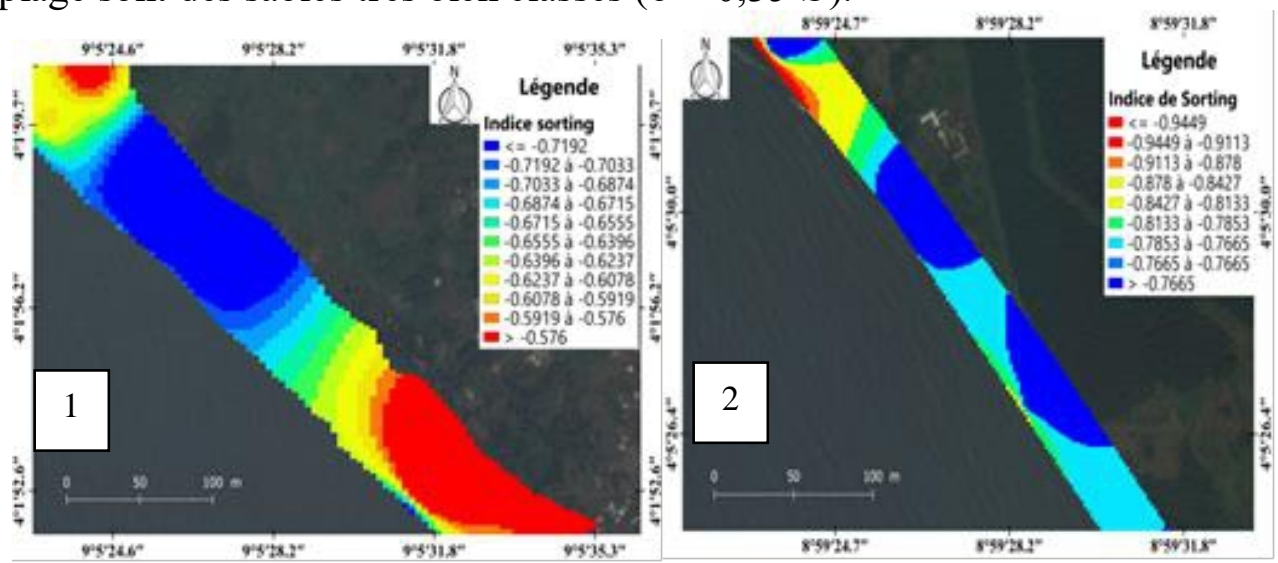

Figure 4. Evolution du coefficient de classement au niveau de la plage de Batoke (1) et au niveau de la plage de Debundscha (2). 


\section{Coefficient de dissymétrie (Sk-Skewness)}

Les valeurs obtenues à Batoke (Tableau 2) sont comprises entre - $0,29 \varnothing$ et $0,126 \varnothing$ sur le haut de plage, entre $0,001 \varnothing$ et $0,246 \varnothing$ sur l'estran et entre $0,329 \varnothing$ et $-0,168 \varnothing$ sur le bas de plage. Ces résultats montrent que les sédiments de la plage de Batoke présentent une distribution granulométrique majoritairement presque symétrique vers les fins $(-0,10 \varnothing<$ Ski $<0,10 \varnothing)$ sur presque toute la plage néanmoins on observe des distributions granulométriques asymétrique vers les fins $(0,10 \varnothing<\mathrm{Ski}<0,30 \varnothing)$ et asymétrique vers les grossiers $(-0,30 \varnothing<\mathrm{Ski}<-0,10 \varnothing)$ sur quelques endroits de la plage.

Les valeurs de Debundscha (Tableau 3), sont comprises au haut de plage entre $-0,237 \varnothing$ et $-0,167 \varnothing$, entre $-0,297 \varnothing$ et $-0,153 \varnothing$ sur l'estran et entre $-0,440 \varnothing$ et- $0,230 \varnothing$ sur le bas de plage. Ces résultats montrent que les sédiments de cette plage présentent une distribution granulométrique asymétrique vers les grossiers sur toute la plage $(-0,30 \varnothing<$ Ski< $-0,10 \varnothing)$.

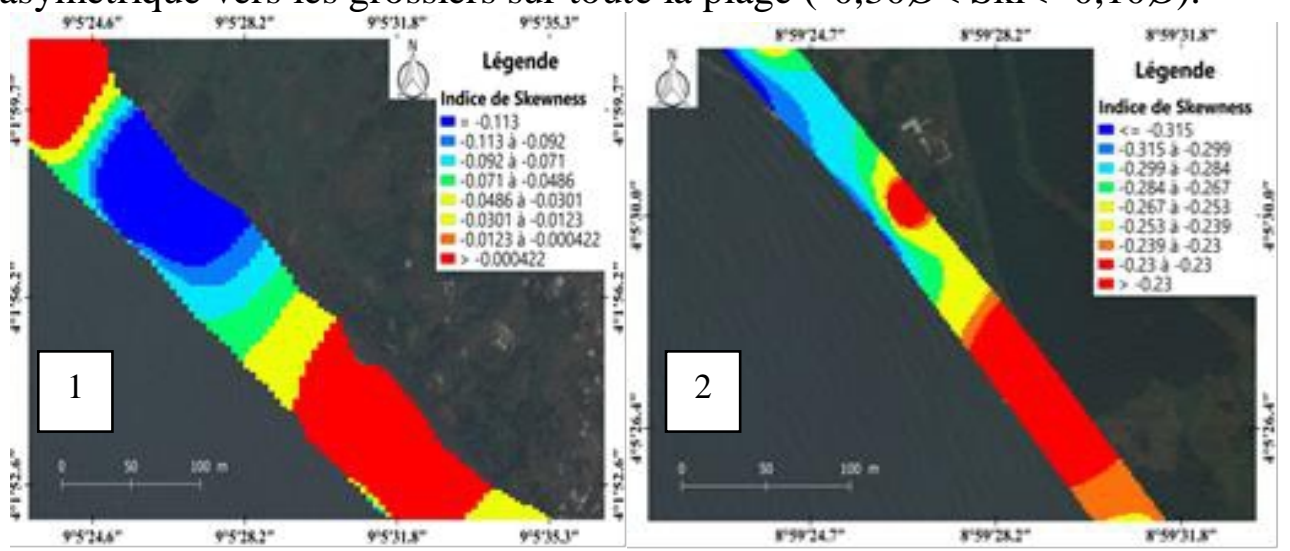

Figure 5. Evolution du coefficient d'asymétrie (Skewness) au niveau de la plage de Batoke (1) et au niveau de la plage de Debundscha (2).

\section{Coefficient d'acuité (K)}

La plage de Batoke (Tableau 2) possède un coefficient d'acuité réparti comme suit : haut de plage (entre 0,918 et 3,12 ) estran (entre 0,949 et 1,413 ) et bas de plage (entre 0,727 et 0,971). Ce résultat $(\mathrm{K}>0)$ signifie que les courbes granulométriques observées sur toutes les unités morphologiques étudiées sont leptokurtiques. Il en est de même des courbes granulométriques des unités morphologiques de la plage de Debundscha (Tableau 3) dont le coefficient d'acuité est reparti de la manière suivante : haut de plage (entre 0,904 et 1,108 ) estran (entre 0,794 et 1,187 ) et bas de plage (entre 0,855 et $1,642)$. 


\section{Coefficient d'uniformité}

Le Coefficient d'uniformité de la plage de Batoke (Tableau 2) est réparti comme suit : haut de plage (entre 1,417 et 1,769), estran (entre 1,5 et 1,619 ) et bas de plage (entre 2,5 et 3 ). Ce résultat montre que le haut de plage et l'estran ont une granulométrie uniforme $(\mathrm{Cu}<2)$ contrairement au bas de plage qui possède une granulométrie variée $(\mathrm{Cu}>2)$.

Celui de la plage de Debundscha (tableau 3) est répartit comme suit : haut de plage (entre 2,143 et 2,5), estran (entre 2,346 et 2,478) et bas de plage (entre 3,35 et 7,011). Ce résultat montre que le haut de plage, l'estran et le bas de plage possèdent tous une granulométrie variée $(\mathrm{Cu}>2)$.

\section{Description des conditions hydrodynamiques en fonction de la distribution spatiale des particules sédimentaires dont les plages de Batoke et Debundscha sont exposées}

Les courbes cumulatives semi-logarithmiques des sédiments des plages de Batoke et Debundscha ont, en général, la forme d'un "S". Ceci indique que ce sont des sables transportés dans un milieu plus au moins agité avec une évacuation des particules fines vers le large par les courants de retour. Le stock sédimentaire est homogène et l'énergie est adaptée à la charge transportée (Atoui \& Brahim, 2009).

Les résultats obtenus précédemment permettent de décrire les conditions hydrodynamiques qui ont permis la distribution granulométrique des sédiments des plages étudiées: La moyenne montre que la plage de Batoke possède à majorité des sables fins tandis que celle de Debundscha possède majoritairement des sables moyens; ceci renseigne sur le fait que l'énergie du courant avec laquelle les sédiments de la plage de Batoke ont été déposés était faible tandis que l'énergie du courant à Debundscha était forte. D'après les résultats du sorting les plages de Batoke et de Debundscha possèdent des sédiments très bien classés. Ceci témoigne que l'environnement de dépôt principal est la plage et que le milieu est peu agité.

Les résultats du skewness ont montré que la distribution granulométrique du classement est presque symétrique sur la plage de Batoke ce qui traduit alors que les sédiments de cette plage ont été transportés par un courant faible qui a favorisé un bon classement des sables fins. Quant à la plage de Debundscha, le classement est asymétrique vers les grossiers avec un skewness inférieur à 1 sur toutes les unités morphologiques de la plage. Ceci signifie que les sédiments de cette plage ont été transportés par un courant faible.

Les données du coefficient d'acuité montrent que les grains au sein de ces deux plages sont bien classés avec des valeurs de $\mathrm{K}>0$ qui montrent que les sédiments ont été transportés et déposés avec une puissance constante de la houle. 


\section{Origine des sédiments des plages étudiées}

Le diagramme des indices granulométriques (Friedman, 1961) des différents sites montre que les sédiments de la plage de Batoke (Figure 6(1)) proviennent à la fois du milieu marin et des apports des rivières tandis que, les sédiments de la plage de Debundscha (Figure 6(2)) proviennent uniquement du milieu marin.

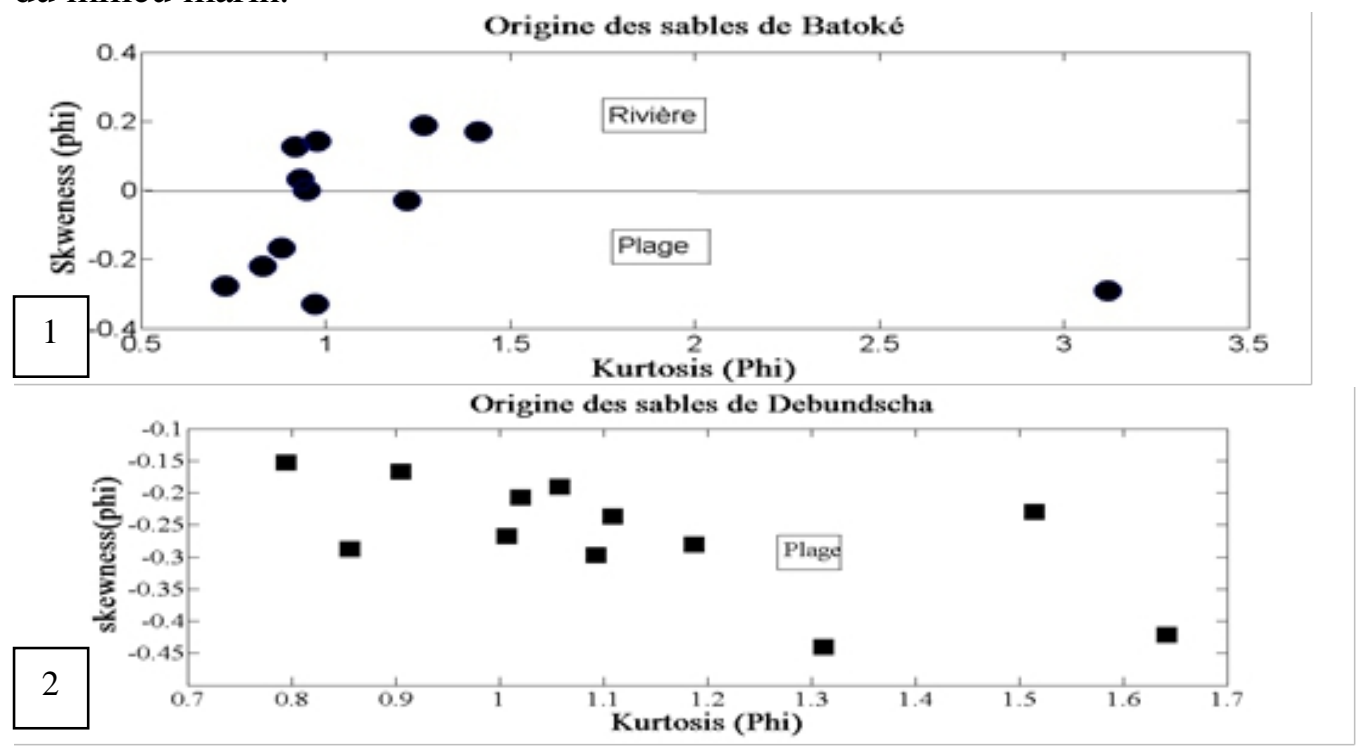

Figure 6. Origine des sédiments des différentes plages

D'après les résultats obtenus, les sédiments des plages de Batoke et Debundscha sont majoritairement transportés par les houles dont les courants sont très faibles mais par rapport au site de Batoké, Debundscha présente des conditions de houle plus importante. Ceci amène à déterminer d'autres paramètres responsables de l'édification morphologique du rivage.

\section{Caractérisation des paramètres de houles et de marées}

Les données de la houle et de la marée du mois d'avril et de mai de la zone d'étude ont étés analysées et synthétisées dans cette partie dont le but est de chercher d'autres paramètres responsables de la distribution granulométrique des sédiments superficiels.

\section{Conditions de houle}

\section{Direction}

La figure ci-dessous nous montre les différentes directions de houle observées pendant la période de l'étude. Ainsi, pendant la période d'étude on a recensé 5 types de houle en fonction de la direction : la direction Sud (S) dominante avec 62,29\%, la direction Sud-Sud-Ouest (SSW) secondaire avec $25,82 \%$, puis suit la direction Sud-Ouest (SW) avec 7,947\%, ensuite la 
direction Ouest (W) avec 1,98\% et enfin la direction Ouest-Sud-Ouest (WSW) avec $1,32 \%$.

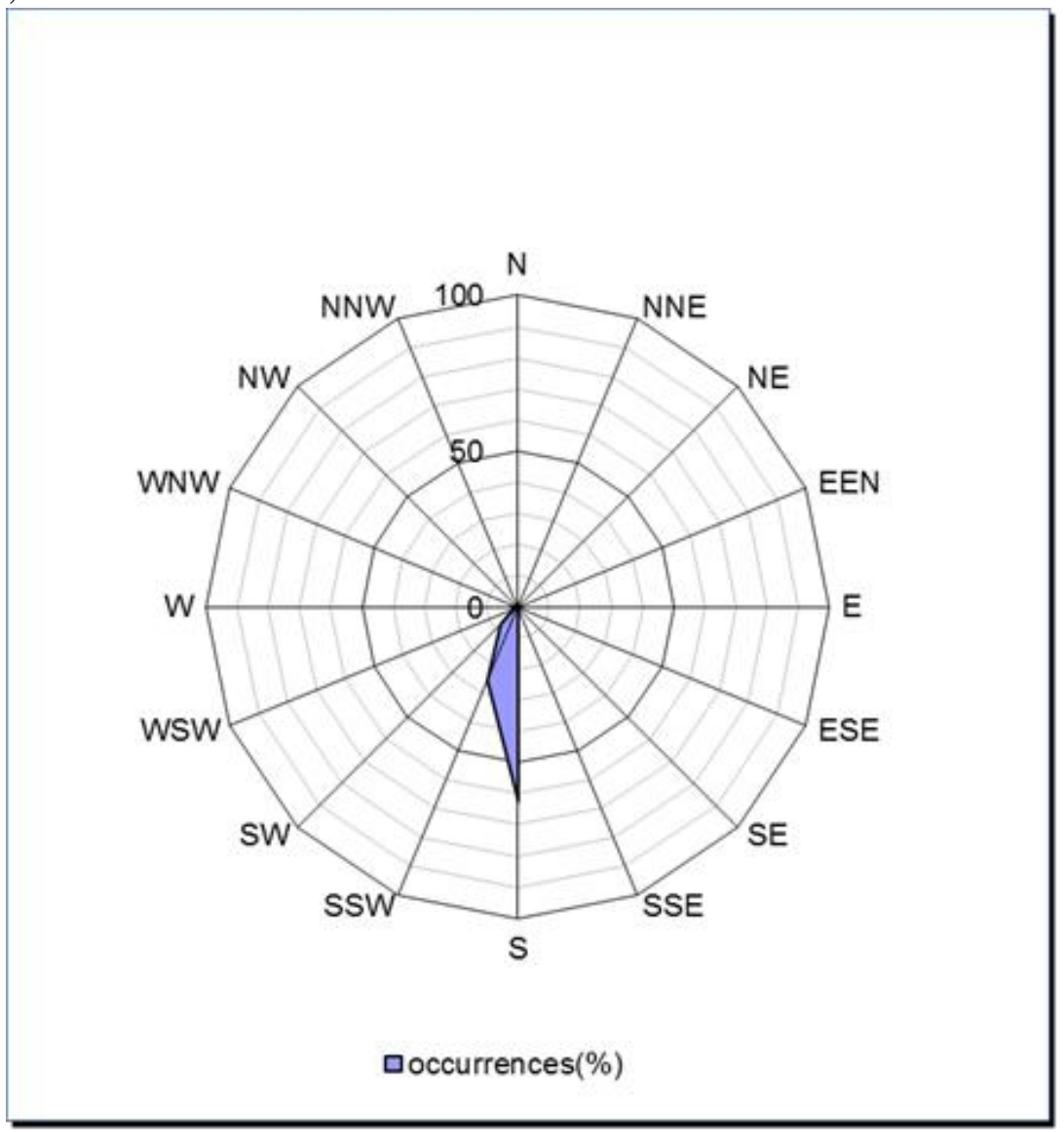

Figure 7. Direction générale de la houle du mois de d'avril et mai

\section{Hauteur significative (Hs)}

La hauteur significative moyenne est de $0,265 \mathrm{~m}$ montrant que la mer est belle dans la plupart des cas pendant la période de l'étude selon la classification de Douglas (Figure 8).

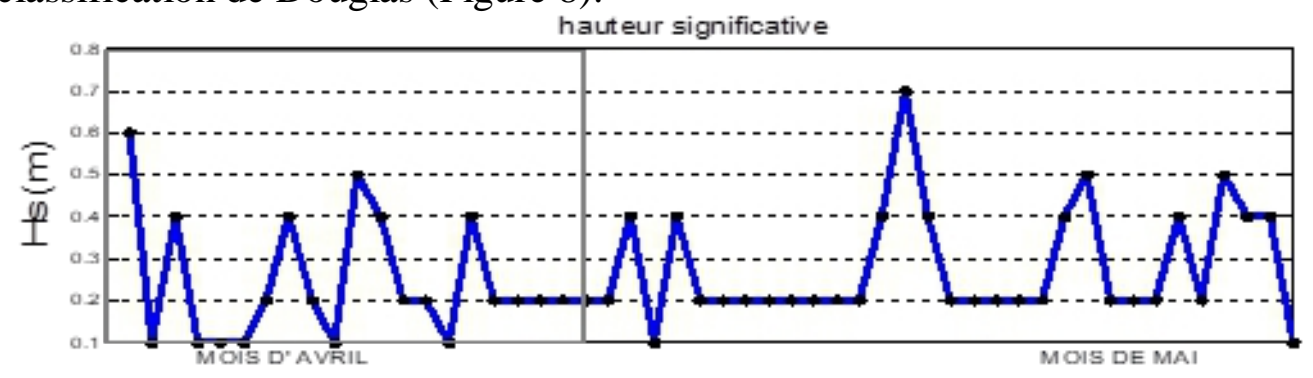

Figure 8. Hauteur significative générale des mois d'avril et mai 
Les données de hauteurs significatives confirment les résultats obtenus par les analyses des indices granulométriques faisant état de ce que le site d'étude est exposé par des houles de courant et d'énergie faibles.

\section{Conditions de la marée}

\section{Marnage}

La marée est de type semi-diurne avec un marnage maximal de 2,3 $\mathrm{m}$ et minimal de 1,1 m enregistré au cours des deux mois de collecte de données. La valeur moyenne du marnage est de $1,752 \mathrm{~m}$ inferieur à $2 \mathrm{~m}$ caractérisant ainsi une plage micro-tidale selon Stepanian (2002).

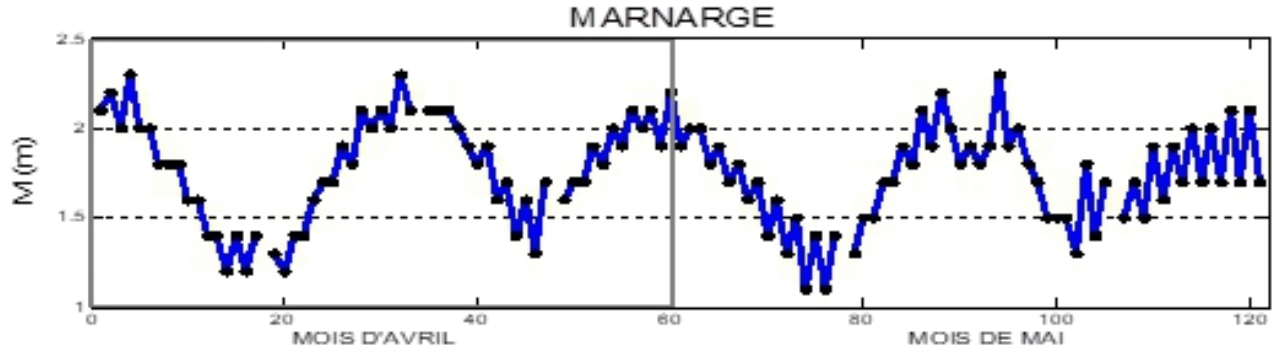

Figure 9. Marnage générale des mois d'avril et de mai

\section{Paramètre de marnage}

Le paramètre de marée s'écrit :

$\mathrm{RTR}=\mathrm{TR} / \mathrm{H}_{\mathrm{b}}$ Où $\mathrm{TR}=$ marnage moyen et $\mathrm{H}_{\mathrm{b}}=$ hauteur des vagues au déferlement estimée en utilisant la formule empirique développée par Komar et Gaughan (1972), exprimée par : $\mathrm{H}_{\mathrm{b}}=\mathrm{H}_{\mathrm{S}}\left[0.563 /\left(\mathrm{H}_{\mathrm{S}} / \mathrm{L}_{0}\right)^{0.2}\right]$ avec $\mathrm{H}_{\mathrm{S}}$ et $\mathrm{L}_{0}$ respectivement hauteur significative et longueur d'onde de la houle au large $\left(\mathrm{L}_{0}=\left(\mathrm{gT}^{2}\right) / 2 \pi\right)$, T est période de la houle et $\mathrm{g}=9,81 \mathrm{~m} / \mathrm{s}^{2}$. Il permet de montrer le paramètre de dominance entre la marée et la houle. Les résultats sont consignés dans le tableau ci-dessous :

Tableau 4. Données mesurées et estimées utilisées dans le calcul du paramètre de marnage

\begin{tabular}{ccccccc}
\hline Sites & $\begin{array}{c}\text { Hs moyen } \\
(\mathbf{m})\end{array}$ & Hb moyen $(\mathbf{m})$ & $\begin{array}{c}\text { Lo moyen } \\
(\mathbf{m})\end{array}$ & $\begin{array}{c}\text { Marnage } \\
\text { moyen }(\mathbf{m})\end{array}$ & RTR & T(s) \\
\hline Batoké & 0,265 & 0,584 & 244,28 & 1,76 & 3,01 & 12,29 \\
\hline Idenau & 0,265 & & & & \\
\hline
\end{tabular}

En évaluant le paramètre de marnage (RTR), une valeur de 3,01 est trouvée donc supérieure à 1 ce qui montre que la marée a une influence dominante sur la houle rencontrée au large de Limbe.

\section{Discussion}

La plage de Batoke est majoritairement caractérisée par des sédiments de la classe des sables fins, très bien classés et dont la distribution est presque symétrique vers les fins; ce résultat va de pair avec celui obtenu par Ismail et 
al en 2015 sur la caractérisation granulométrique et minéralogique des sédiments littoraux : dynamique sédimentaire et sources de matériaux réalisée sur la baie d'Agadir (Maroc Atlantique). Ceux-ci déduisent que le milieu est peu agité ceci dû au transport et dépôt des sédiments du fait d'un hydrodynamisme faible de la houle. La plage de Debundscha quant à elle est caractérisée par une prédominance des sédiments de la classe des sables moyens, très bien classés et dont la distribution est asymétrique vers les grossiers ; ce résultat est identique à celui obtenu par N'Goran et al en 2011 sur l'étude sédimentologique et esquisse paleoenvironnementale des formations de fresco sur le littoral ivoirien situé dans le Golfe de Guinée au même titre que la côte camerounaise. Ces derniers montrent qu'à l'issu des analyses granulométriques effectuées, les plages sont recouvertes de sables très bien classés. Il en ressort donc que les dépôts sédimentaires étudiés sont issus d'une réduction progressive de l'énergie de l'agent de transport.

\section{Conclusions et Perspectives}

$\mathrm{Au}$ terme de cette étude, de nombreuses connaissances ont été acquises, elles ont permis de faire un état sur les paramètres hydrodynamiques agissant sur la côte ouest de Limbe. Il était question pour cette étude de contribuer à la détermination de l'importance des paramètres hydrodynamiques dans la répartition spatiale des sédiments des plages à Limbe. Les analyses granulométriques menées ont permis de montrer une forte relation entre l'hydrodynamisme du milieu et la répartition des grains. Par ailleurs l'évaluation du paramètre de marnage montre que la marée pourrait être le paramètre édificateur majoritairement responsable de la répartition spatiale des sédiments sur ces deux plages.

Cette étude a été entreprise dans le but de renseigner la population sur l'état de la mer et sur les différents paramètres hydrodynamiques pouvant conditionner le dépôt et la classification des sédiments sur la côte de Limbe, Acquérir des techniques sur l'analyse des paramètres granulométriques des sédiments et l'interprétation des paramètres hydrodynamiques en fonction de cette dernière, participer à la protection de la population par la détermination des zones à risques auxquelles elle peut être soumise inconsciemment sur la côte de Limbe, pouvoir gérer le littoral, les populations qui y vivent et les infrastructures qui s'y trouvent et constituer une banque de données pour la recherche scientifique. En guise de perspective, une analyse des paramètres hydrodynamiques responsables de la morphologie de toutes les plages de Limbé et de la côte ouest-camerounaise doit être entrepris par les chercheurs et aussi une analyse minéralogique des sédiments de toutes les plages de la cote-Ouest doit être entrepris pour détermination l'origine des sédiments. 


\section{Remerciements}

Les auteurs souhaitent remercier le personnel de la Station Spécialisée de Recherche Halieutique et Océanographique de Limbé au Cameroun pour l'utilisation de leurs installations et leur soutien logistique sur le terrain.

\section{References:}

1. Aagaard, T. (2014). Sediment supply tobeaches: Cross-shore sand transport onthe lower shoreface. J. Geophys. Res. Earth Surf. n¹19, PP. 913-926, doi:10.1002/2013JF003041

2. Abuodha, J.O.Z. (2003). Grain size distribution and composition of modern dune and beach sediments, Malindi bay coast, Kenya. Journal of African Earth Sciences n³6 pp. 41-54

3. Adjoussi, P. (2001). Impacts du prélèvement du sable marin sur l'évolution du trait de côte à Yoff : essai d'étude de vulnérabilité. Mémoire de D.E.A de géographie, Université Cheikh Anta Diop, Dakar, 72 p.

4. Atoui, A. \& Brahim, M. (2009). Distributions granulométriques des sédiments de surface de la frange littorale du Ras Taguermess au Ras Marmour (golfe de gabes, Tunisie). Conférence Méditerranéenne Côtière et Maritime Edition 1, Hammamet, Tunisie.

5. Biagi, P. (1993). Soil exploitation and early agriculture in northern Italy. N³, pp.164-168. https://doi.org/10.1177/095968369300300208

6. Bird, C. (1985). Coastline changes a global review. John Wiley \& Sons Chichester, New York, Brisbane, Toronto, Singapore, 219 p.

7. Chauhan, O.S. (1992). Laminae and grain-size measures in beach sediments, East Coast Beaches. Indian J. Coastal Res. n`8 pp. 172182.

8. Folk, R.L. (1966). A review of grain size parameters. Sedimentology. n 6 pp. 73-93

9. Folk, R. \& Word, W. (1957). Brazors river bors, a study in significance of grain size parameters. J. Sedim. Petrol., n²7, pp. $13-27$.

10. Ismail, A., Lahcen, D., Fatim, Z.I., \& Brahim, O. (2015). Caractérisation minéralogique et granulométrique des sédiments littoraux de la baie d'Agadir (Maroc atlantique) : dynamique sédimentaire et sources des matériaux, J.Mater. Sci. n7 pp. 37113723.

11. Komar, P.D. \& Gaughan, M. K. (1972). Airy Wave Theory and Breaker Height Prediction. In: Proceedings of the 13th conference on coastal engineering, Vancouver, 10-14 July 1972. ASCE, New York, pp 405-418 
12. Muller, J.P. \& Gavaud, M. (1979). Atlas de la République unie du Cameroun, édition jeune Afrique, Paris, pp 25-26.

13. N'goran, J.P., Zéli, B.D., Sylvain, M., Akoua, C.K., Kouamé, A., N'Zalassé B., Yassi, J.T., Yaba, L.H.K., \& Kouamé, L.D. (2011). Etude sédimentologique et esquisse paleoenvironnementale des formations de fresco. Sciences \& Nature, $\mathrm{N}^{\circ} 1$, pp. $73-84$.

14. Nordstrom, K.F. (1977). The use of grain size statistics to distinguish between high and moderate energy beach environments. J. Sedim. Res. $n^{\circ} 47$ pp. 1287-1294.

15. Paskoff, R. (2001). Les littoraux, Impact des aménagements sur leur évolution. Paris, Armand Colin, 264 p. 